\title{
Gastric tuberculosis presenting with a huge abdominal mass
}

Jun Uk $\underline{\mathrm{Lim}}^{1}$, MD, Yee Hyung $\underline{\mathrm{Kim}}^{2}$, MD, Cheon Woong $\underline{\mathrm{Choi}}{ }^{2}$, MD, Jong Hoo $\underline{\mathrm{Le}}^{3}$, MD

\begin{abstract}
Tuberculosis of the stomach is extremely rare. We report the case of a 38-year-old woman who presented with epigastric discomfort and a palpable mass that persisted for a period of one month. We also report our findings from the abdominal computed tomographic, upper endoscopic and endoscopic ultrasonographic examinations of the patient. Abdominal computed tomography (CT) showed the presence of a large mass with an irregularly contoured low attenuation lesion. Upper endoscopy and endoscopic ultrasonography revealed a protruding ulcerative mass with an ill-defined heteroechoic subepithelial lesion originating from the gastric submucosal layer. This was previously misdiagnosed as a gastrointestinal stromal tumour. Endoscopic biopsy specimen was positive on acid-fast bacillus staining, and polymerase chain reaction for Mycobacterium tuberculosis was also positive. Abdominal CT and endoscopy at the patient's three-month follow-up showed near complete resolution of the lesion.
\end{abstract}

Keywords: abdominal mass, endoscopic ultrasonography, stomach, tuberculosis

\section{INTRODUCTION}

The gastrointestinal tract is the sixth leading site of extrapulmonary tuberculosis (TB). Gastric TB is, however, extremely rare in patients with pulmonary TB; gastric TB as the primary or isolated involvement in immunocompetent hosts is even rarer. ${ }^{(1,2)}$ Since this disease resembles peptic ulcer disease and mimics gastric malignancy, its diagnosis requires clinical, histological and/or microbiological confirmation. . $^{(3,4)}$ Herein, we report a case of gastric TB presenting with a huge, palpable abdominal mass that was managed with medical treatment.

\section{CASE REPORT}

A 38-year-old woman was referred to our institution for evaluation of an abdominal mass. She complained of epigastric discomfort that persisted for about one month. This discomfort was not associated with nausea or vomiting. She did not have a fever nor did she experience any weight loss. Physical examination was unremarkable except for the presence of a palpable abdominal mass in the epigastrium. The patient was previously on medication for pulmonary TB. However, her adherence to medication was poor. Her previous treatment regimen included isoniazid, rifampicin and ethambutol.

Chest radiography showed persistent cavities, with a thick wall in the upper and lower lobes of the right and left lungs, respectively. Sputum acid-fast bacillus (AFB) stain and culture on liquid and solid media were negative at the time of the patient's presentation to the hospital. The patient's complete blood cell count was unremarkable, her hepatic and renal functions were normal, and she was negative for HIV infection. Standard abdominal radiography was also unremarkable. However,

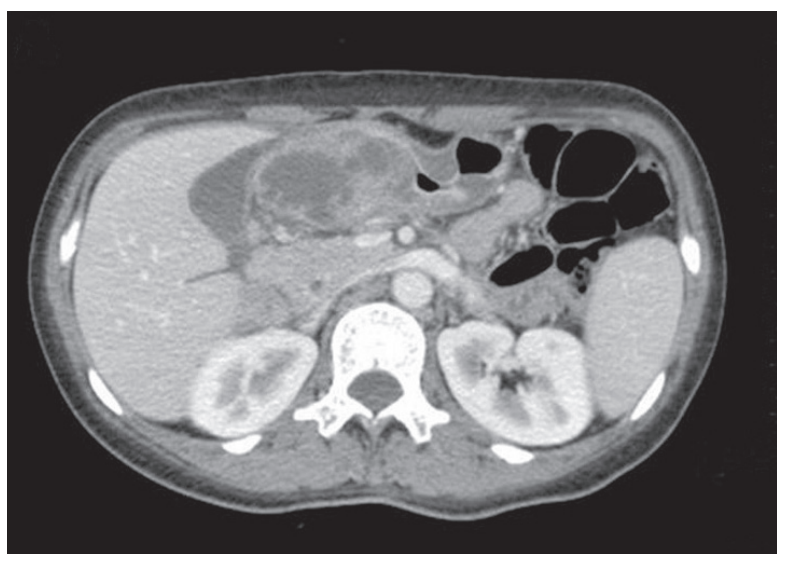

Fig. 1 Abdominal CT shows an irregular, lobulating mass with low attenuation, measuring about $7.5 \mathrm{~cm} \times 5.0 \mathrm{~cm}$, located between the antrum of the stomach and the transverse colon.

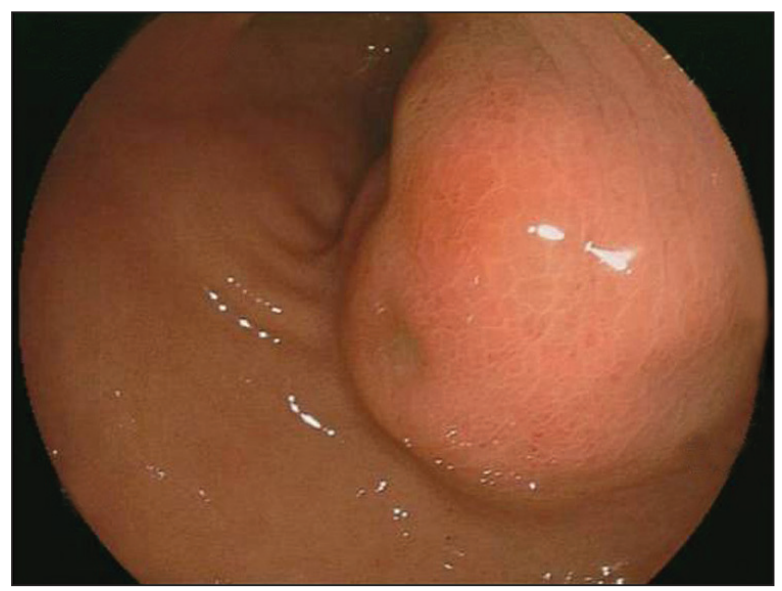

Fig. 2 Gastroduodenal endoscopy shows an intraluminal protruding mass lesion measuring about $4.0 \mathrm{~cm} \times 4.0 \mathrm{~cm}$, with a $1.5-\mathrm{cm}$ overlying superficial ulceration in the posterior wall of the gastric antrum.

${ }_{1}^{1}$ Department of Gastroenterology and Hepatology, ${ }^{2}$ Department of Pulmonary and Critical Care Medicine, Kyung Hee University Hospital at Gangdong, School of
Medicine, Kyung Hee University, Seoul, ${ }^{3}$ Department of Pulmonary and Critical Care Medicine, Jeju National University Hospital, Jeju, Republic of Korea
Correspondence: Dr Yee Hyung Kim, Assistant Professor and Consultant, Department of Pulmonary and Critical Care Medicine, Kyung Hee University Hospital at Correspondence: Dr Yee Hyung Kim, Assistant Professor and Consultant, Department of Pul
Gangdong, 149 Sangil-dong, Gangdong-gu, Seoul 134-727, Republic of Korea. kumc92@naver.com 

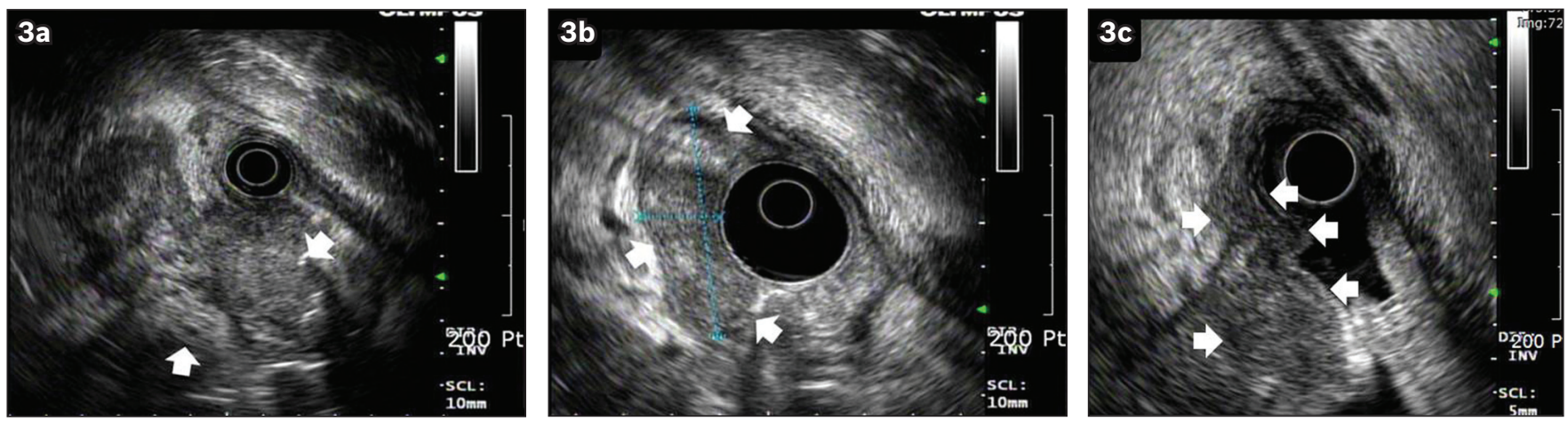

Fig. 3 Endoscopic ultrasonography images show an ill-defined, heterogeneous echoic lesion, measuring $8.0 \mathrm{~cm} \times 5.0 \mathrm{~cm}$, originating from the gastric submucosal layer (white arrows).
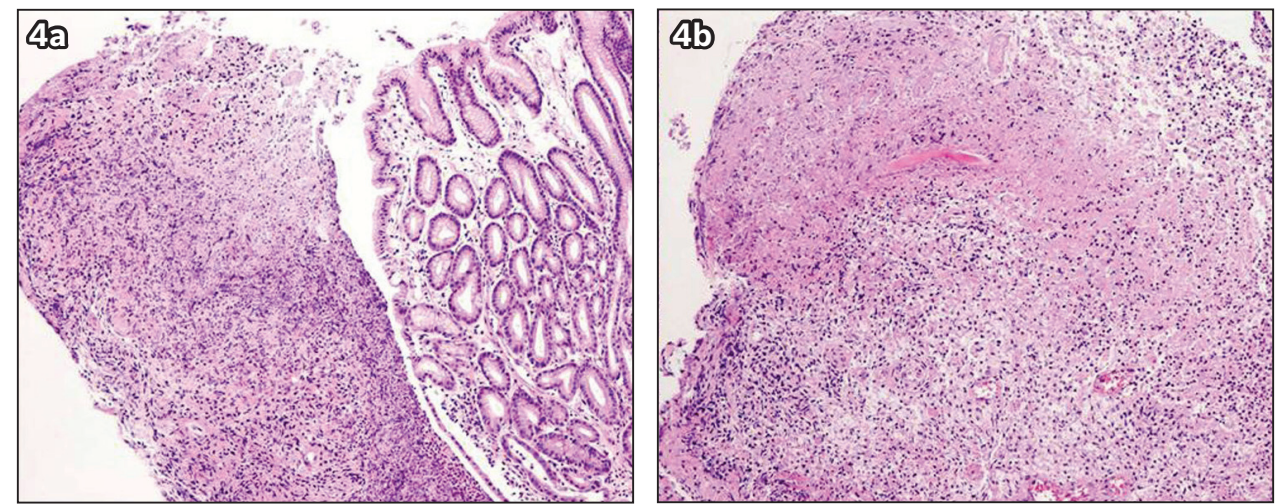

Fig. 4 Photomicrographs of the stomach biopsy specimen show (a) ulceration with patchy necrotic areas (Haematoxylin \& eosin, × 100); (b) the ulcer detritus, which has ill-defined suppurative granulomatous lesion with caseous necrosis. However, multinucleated giant cells were not noted in this specimen (Haematoxylin \& eosin, $\times 100)$.

abdominal computed tomography (CT) revealed an irregularly contoured, low attenuation lesion measuring about $7.5 \mathrm{~cm} \times 5.0 \mathrm{~cm}$ around the gastrocolic ligament, abutting the gastric antrum and transverse colon (Fig. 1). Gastric antral wall thickening and lymphadenopathy were also seen.

Upper endoscopy revealed the presence of an intraluminal protruding mass lesion measuring about $4.0 \mathrm{~cm} \times 4.0 \mathrm{~cm}$, with a $1.5-\mathrm{cm}$ overlying superficial ulceration, in the posterior wall of the gastric antrum (Fig. 2). When the mass was compressed by a biopsy forcep, the consistency of the mass was found to be somewhat hard. No rolling sign was observed. Thick, whitish, cheese-like necrotic material was observed at the base of the ulcer. Additionally, endoscopic ultrasonography (EUS) demonstrated an ill-defined heteroechoic subepithelial lesion measuring about $8.0 \mathrm{~cm} \times 5.0 \mathrm{~cm}$ and originating from the gastric submucosal layer (Fig. 3). The perigastric lymph nodes were slightly enlarged. Diagnoses such as gastrointestinal stromal tumour (GIST), unliquefactioned gastric wall abscess and leiomyoma were the endoscopist's primary suspicions. Endoscopic biopsy performed on the ulcer base revealed an ill-defined necrotic granuloma with an abscess (Fig. 4). Immunohistochemical stains for C-Kit, desmin and S-100 were negative. However, AFB stain was strongly positive. Nested polymerase chain reaction for Mycobacterium tuberculosis was also positive.
The patient was ultimately confirmed to have a tuberculous abscess, which originated from the gastric wall and fistulated into the stomach, complicated by insufficient treatment. Due to the concern of acquired resistance to first-line anti-TB drugs, an attending physician prescribed a new regimen for the patient's gastric TB. The regimen included four second-line anti-TB drugs (i.e. kanamycin, moxifloxacin, prothionamide and cycloserin) and pyrazinamide. Abdominal CT obtained three months after the start of therapy showed a marked decrease in the size of the gastric wall mass, which involved the gastrocolic ligament (Fig. 5). Physical examination revealed no remaining palpable abdominal mass. Follow-up endoscopy after three months demonstrated a considerably reduced polypoid mass lesion and the active ulcer progressed to a healing stage ulcer (Fig. 6). The patient was considered free from TB after 12 months of treatment.

\section{DISCUSSION}

Even in parts of the world where intestinal TB is common, involvement of the stomach in both primary and secondary TB is considered rare. ${ }^{(5,6)}$ The ileocecal region is the most common site of intra-abdominal TB. ${ }^{(5)}$ The reasons for the rarity of gastric TB include the bactericidal property of gastric acid, the scarcity of lymphoid tissue in the gastric wall and the protective gastric mucosa of the stomach. ${ }^{(7)}$ Gastric TB 


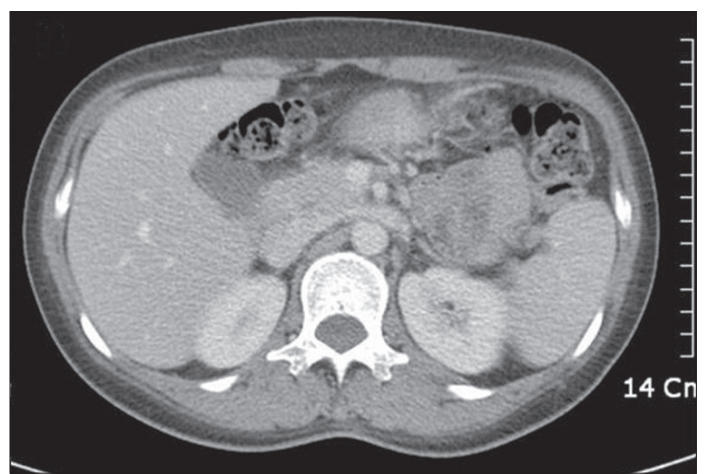

Fig. 5 Abdominal CT after three months of anti-tuberculous medication shows a marked decrease in the size of the gastric wall mass, which involves the gastrocolic ligament.

commonly mimics peptic ulcer disease or malignancy. A study by Rao et al found gastric outlet obstruction (61\%) and gastrointestinal bleeding (26\%) to be the major presenting features of the disease. ${ }^{(3)}$ At times, however, gastric TB exhibits no specific signs or symptoms, and no characteristic endoscopic or radiographic features. Therefore, diagnosis requires a high index of suspicion and histological or microbiological study. In spite of the presence of a huge, palpable abdominal mass, our patient did not exhibit any specific signs or symptoms associated with obstruction or bleeding. On endoscopy, our initial gross impression included GIST, gastric wall abscess, gastric cancer and leiomyoma; even a trained endoscopist did not consider the possibility of gastric TB. Due to the rarity of gastric TB, few reports have analysed the characteristics of gastric TB on EUS. ${ }^{(8)}$ Although we described an EUS finding of TB invasion of the gastric wall in the case of our patient, this finding was not sufficiently specific to exclude other tumours and abscesses. Our findings support the fact that gastric TB does not have a characteristic appearance on endoscopy, and direct inspection of the lesion has a limited role in its diagnosis. As a result, a diagnosis of gastric TB can only be made by histological and/or microbiological study, coupled with a high index of suspicion.

Elective surgery should be reserved for complications such as obstruction, fistula formation, or intractable ulceration. ${ }^{(3)}$ In the present case, we decided to start the patient on anti-TB medication without surgery soon after final diagnosis because despite the large size of the lesion, she did not have nausea

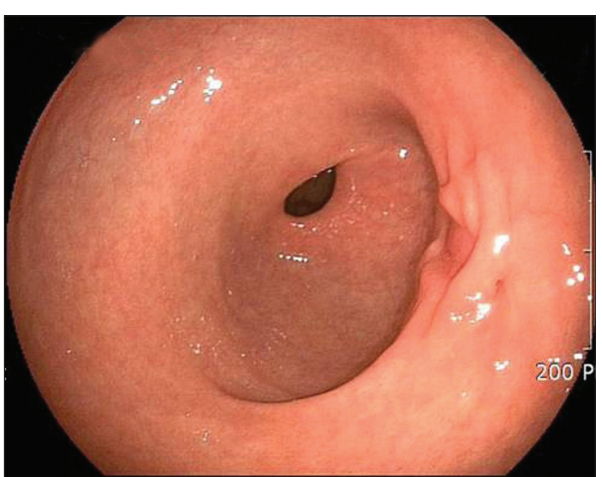

Fig. 6 Gastroduodenal endoscopy after three months of therapy shows disappearance of the protruding mass and the presence of a scar (from healing).

nor was she vomiting. Upper endoscopy and abdominal CT performed at the patient's three-month follow-up showed dramatic improvement.

This report describes a case of TB invading the gastric wall, forming a huge intra-abdominal mass. Regardless of the size of the mass, gastric TB can be managed solely with medical treatment if there are no complications such as obstruction, bleeding or fistula. Since findings on upper endoscopy and EUS are not specific, a high index of suspicion and histological and/or bacteriological evaluation are important in rendering the diagnosis of gastric TB.

\section{REFERENCES}

1. Singh B, Moodley J, Ramdial P, et al. Primary gastric tuberculosis. A report of 3 cases. S Afr J Surg 1996; 34:29-32.

2. Subei I, Attar B, Schmitt G, Levendoglu H. Primary gastric tuberculosis: a case report and literature review. Am J Gastroenterol 1987; 82:769-72.

3. Rao YG, Pande GK, Sahni P, Chattopadhyay TK. Gastroduodenal tuberculosis management guidelines, based on a large experience and a review of the literature. Can J Surg 2004; 47:364-8.

4. Di Placido R, Pietroletti R, Leardi S, Simi M. Primary gastroduodenal tuberculous infection presenting as pyloric outlet obstruction. Am J Gastroenterol 1996; 91:807-8.

5. Abrams JS, Holden WD. Tuberculosis of the gastrointestinal tract. Arch Surg 1964; 89:282-93

6. Subei I, Attar B, Schmitt G, Levendoglu H. Primary gastric tuberculosis: a case report and literature review. Am J Gastroenterol 1987; 82:769-72.

7. Bradyopadhyay SK, Brandyopadhyay R, Chatterjee U. Isolated gastric tuberculosis presenting as haematemesis. J Postgrad Med 2002; 48:72-3.

8. Puri R, Sud R, Kumar M, Bhagat S, Khaliq A. Tubercular gastroduodenal abscess presenting with obstructive jaundice: diagnosis with EUS-guided FNA. Gastrointest Endosc 2011; 74:1400-1. 